\title{
Developing TAMEx Model For Availability Aspect of E-Exam Security in WLAN Environment
}

\author{
Gede Sukadarmika ${ }^{1}$, Rukmi Sari Hartati ${ }^{2}$ Linawati $^{3}$, Nyoman Putra Sastra ${ }^{4}$, A.A.N. Amrita \\ Department of Electrical Engineering, Faculty of Engineering, Udayana University, Bali, Indonesia \\ sukadarmika@unud.ac.id \\ 2,3,4,5) Department of Electrical Engineering, Faculty of Engineering, Udayana University, Bali, Indonesia
}

\begin{abstract}
The internet has provided tremendous impact in educational development throughout the world. E-Exam system is one of educational component which has been used increasing dramatically for evaluating educational process. As an e-exam feature, uniformity of system access and time duration of the exam to all examinees is very important to be considered. Exam manager usually utilizes a fixed network to connect all e-exam terminal in order to maintain service stability on e-exam systems. However, the use of a fixed network will be difficult to be implemented if the number of examines is large. There are not many Education institutions have adequate ICT infrastructure or e-exam terminals for all students. Most institutions develop WLAN networks as an alternative to being able to provide ICT services to all students. With the development of mobile device technology (Handphone or Laptop) that strongly supports the learning process through mobile devices. This research aims to develop application of the Time Adaptive for Mobile E-Exam (TAMEx) Model. It serves to maintain the reliability of e-exam implementation on WLAN networks. The possible connection disruptions is anticipate by seeking time compensation only to participants who has experienced the connection problem. The time compensation must be accordance to the duration of the occurrence of connection loss. Application development has been carried out and is functioning properly. The report of application shows that it has succeeded in identifying the connection disturbance duration and provide time compensation to the proper examinee.
\end{abstract}

Index Terms - availability, e-exam, security, TAMEx, WLAN.

\section{INTRODUCTION}

$\mathrm{T}$ HE Time Adaptive for Mobile e-Exam (TAMEx) Model is a conceptual framework to support the implementation of e-exams through WLAN networks and the Bring Your Own Devices (BYOD) mechanism [1]. The implementation of the TAMEx model can be an alternative solution for the implementation of e-exam for institutions that have limited ICT infrastructure and e-exam terminal devices. Thus, the application of e-exam becomes more flexible with regard to space and institutions do not need to prepare e-exam terminals as much as the number of examinees.

The availability of information on e-exam system should be of particular concern when the system is applied to an ICT infrastructure network whose service quality is less stable for example in wireless environment. This research uses Wireless Local Area Network (WLAN) as network access to e-exam system. As well known that the quality of WLAN service is influenced by various factors such as distance of access point to the user, interference, fading effect, number of users connected with access point and others [2],[3]. However, the use of WLAN is very useful in terms of space flexibility and the development of service coverage. For this purpose, this research is deemed necessary in maintaining the availability of services without disregarding aspects of accountability, fairness, free from fraud, and minimizing proctor in the implementation of e-exam.

The security of e-exam systems is a matter of serious concern in the application of e-learning. At the online exam, the location of the supervisor varies from the location of the examinees. With the increase in distance between the supervisor and the exam, the likelihood of cheating increases. To avoid such situations, examinees should continue to be monitored [4]. Along with the growing use of e-exam in recent decades, the equipment used to present and manage the needs of e-exam must be coupled with an efficient and reliable security mechanism to ensure the system can be declared as one of the most reliable media [5].

Many techniques are proposed to provide security during e-exam implementation. Research on the use of continuous authentication by using biometric security systems has also been done [6],[7],[8]. Various technologies and equipment are also used to ensure that the implementation of e-exam is free from cheating.

Previous research, mostly consent in e-exam security in 
terms of to anticipate e-exam from various fraud that may be done during its implementation. This research however, eexam security is done on its availability aspect.

In the implementation of e-exam, time allocation for conducting examination is one of the most important things for examinee. Usually the time is determined at the start and at the end of the examination. When the exam session begins, the timer will begin to be active, the participants then complete to answer the exam questions in accordance with the time allocated. When the time is up, the system gives warning and the user session $\log$ s is switched off automatically [9].

WLAN plays a very significant role in the provision of network services everywhere to the contemporary society as a result of mobility and the application of wireless networks. But it is a challenge for WLANs to set up higher throughput than wired networks. The robust signal received (RSSI), damping and track loss is highly dependent on the propagation environment. The location of the Access Point placement (AP) has a very significant effect on the quality of WLAN services [10].

This research emphasizes efforts to develop application based on the TAMEx Model in order to maintain the delivery of e-exam content to examinees such that in the event of any disturbance to the quality of WLAN signals, participants can still able to complete their exams without losing their time allocation.

\section{TIME AdAPTIVE FOR MOBILE E-EXAM ModEL}

\section{A. TAMEx Model Desain}

The Time Adaptive for Mobile e-Exam (TAMEx) Model is a framework developed to maintain the reliability of eexam implementation on wireless networks. At this research, TAMEx was developed for the implementation of e-exam on WLAN networks and using laptops as the e-exam terminals. The sequence of process diagrams of the TAMEx Model and flowchart is shown in [1]. In general, the e-exam process is divided into two namely Preparation and examination.

In the preparation process the TAMEx Adapter application is installed on the e-exam terminal, which is a laptop carried by e-exam participants (BYOD). This application functions for the authentication mechanism of participants as well as e-exam terminal devices on the access point. In addition, at the examination stage, this application serves to generate TAMEx data, which is a row of data that contains information about the user's identity and WLAN properties of the e-exam terminal. If the e-exam terminal conditions are connected, TAMEx data is generated and sent periodically to the e-exam server.

The examination process will begin if the authentication of the examinee and e-exam terminal is successful and the delivery of questions by the e-exam server begins. At the same time, the TAMEx adapter also starts generating TAMEx data and sends it to the e-exam server periodically. In normal conditions (no connection problems occur) generation and transmission of TAMEx data is done periodically and continuously. However, if the e-exam terminal experiences a connection failure with an RSSI level $<20 \%$, the generated TAMEx data cannot be sent. When the e-exam terminal receives an RSSI level $\geq 20 \%$ of the TAMEx data is sent again. The TAMEx adapter also calculates the duration of the interrupted error in order to request a time compensation to the server. The e-exam server calculates the duration of the interruption based on the duration between the arrival of the TAMEx Data one and the next data arrival. Furthermore, time compensation is given to e-exam terminals that experience interference only in accordance with the duration of the disturbance they experience. Thus, the duration of the implementation of the e-exam for each examinee will adapt to the quality of the received signal, but the total time spent on conducting the e-exam of each examinee will be the same, which is in accordance to examination time which was setted up by the e-exam management.

\section{B. The TAMEx Data Flow Diagram}

Data Flow Diagrams are used to describe the system that is emphasized to see how the processes that occur from the applied system as well as existing manual processes. By using DFD, the system process can be explained to system users so that it gets clarity in the application of the application. here are discussed, Context level DFD and level 1 DFD are described.

\section{Data Flow Diagram Level Context}

Figure 1 shows a DFD diagram of the developed TAMEx model. It can be seen that there are two (2) entities associated with the e-exam system in this TAMEx model, namely the admin and the examinees (users) with their respective relationships to the system. The database stores and manages two data sources namely e-exam data and user data.

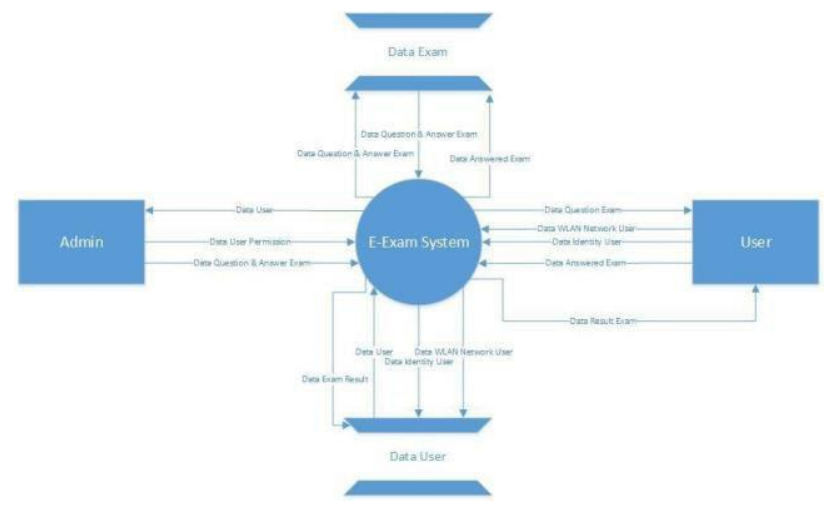

Fig. 1. DFD Level Context of TAMEx Model

Admin entities and user entities when login to the system need to verify based on the user password on the e-exam system. It means that before login in, it is required first to activate and input the user name in the TAMEx Adapter application. This mechanism is intended as a process of authenticating data user. The access as Admin has a role to manage the examination and the role of all users. 
Moreover, for the user entity, the TAMEx model deliver exam questions to the user. While from the system user will receive the answer data of the question, user identity and WLAN data interface of the e-exam terminal. There two kinds of data stored on this system namely Data TAMEx and exam data. The TAMEx Data is generated and sent by eexam terminal. Then, the exam data is the data of e-exam management and e-exam question.

\section{Data Flow Diagram Level 1}

For a more detailed description of the data flow diagram at the context level, the following Data Flow Diagram Level 1 is presented from the TAMEx system, which is shown in Figure 4.5.

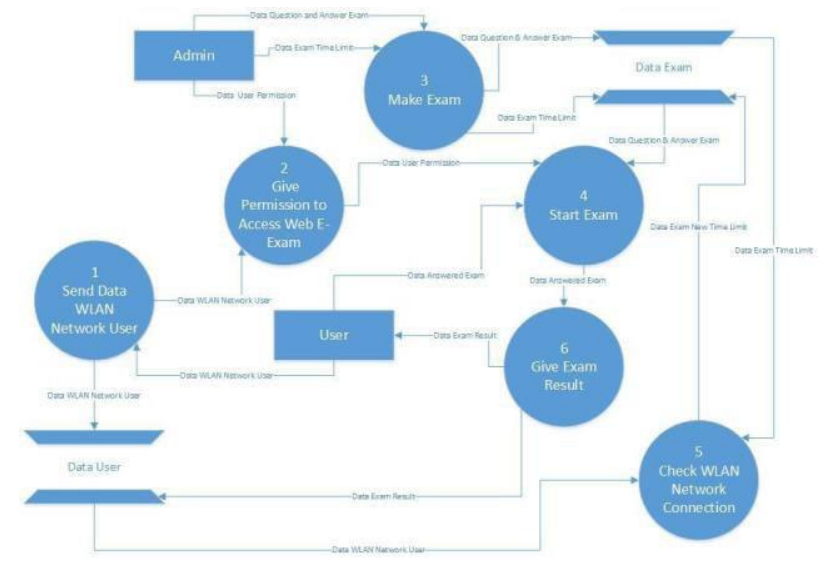

Fig. 2. DFD Level 1

As can be seen from figure 2, there are 6 processes performed on the system, namely:

1. Sending WLAN network interface data from the e-exam terminal.

2. Granting user access by the Admin

3. Admin creates questions and answers to the e-exam system and setting the time of the exam.

4. Starting the exam by exam participants, so the examinee sends the answer data from the given questions.

5. When the exam is complete, the system provides confirmation about the results of the exam to each user.

6. Checking the connection status of the user device based on the data of WLAN network interface when generate TAMEx Data. This data is sent periodically by e-exam terminal. The data sent is stored in the following database with the identity of the time when the data was sent. Furthermore, the system will receive the identity of the time remaining for the implementation of the test.

\section{APPLICATION DISPLAY AND RESUlT}

Here are discussed the display of the application, result of the recorded TAMEx data and record of the given time compensation to the examinee.

\section{A. Application Display}

The application display is presented sequentially according to the stages of the TAMEx model processes. At the preparation stage, the user firstly has to be registered as an examinee at the examination system. It is done by completing the biodata form shown in Figure 3. The initial display of the e-exam application is shown in Figure 3a. Users who have not been registered yet must press the register button which next displays the registration form shown in Figure $3 b$.

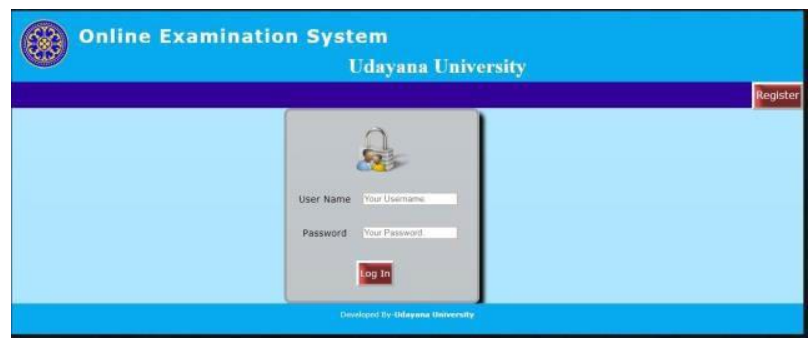

(a)

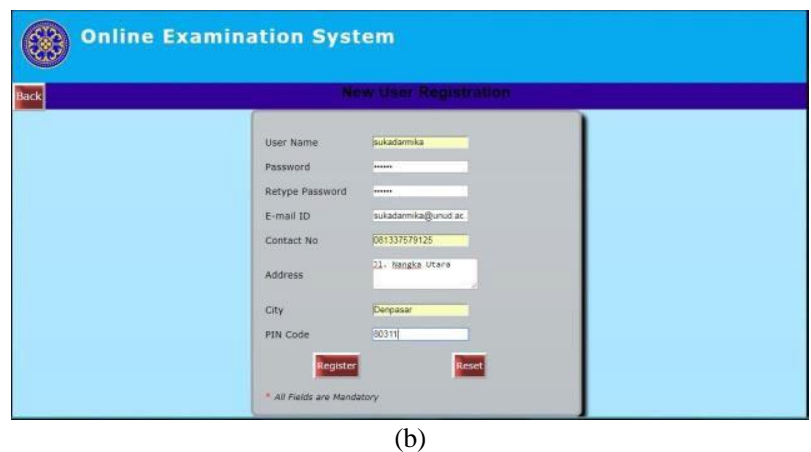

Fig. 3. Registration user at the examination system

(a) The initial display of Examination System

(b) User's registration form

The next stage is the initial preparation for the implementation of e-exam. In order to take the e-exam, the examiners must bring their own laptop device which will be used as the e-exam terminal. On that device, they have to install the TAMEx Adapter application which functions for the authentication and processing of TAMEx Data.

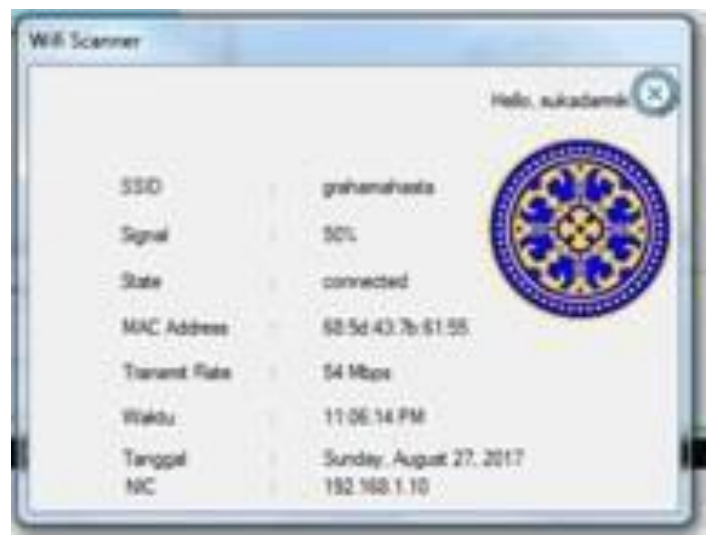

Fig. 4.TAMEX Adapter Activation 
The activation of the TAMEx Adapter application is shown in Figure 4. The user first needs to be authenticated by login using username that entered in accordance with the user name at registration on the e-exam system. As can be seen from the figure that the system can read and generate WLAN interface properties of the e-exam terminal device such as SSID, Signa Strength (RSSI), Connection Status, MAC Address, Data Rate, Time and IP Address.

With the activation of the TAMEx Adapter, the examinee can begin the test process by logging into the e-exam system, then determining the subject exam to be followed. If the subject exam has been selected, then the examinee must enter the code of the test to be followed. This code is delivered by the administrator exam directly to examinees. This mechanism is as a registered examinee authentication to take the appropriate subject test. The application display for this process is shown in Figure 5.

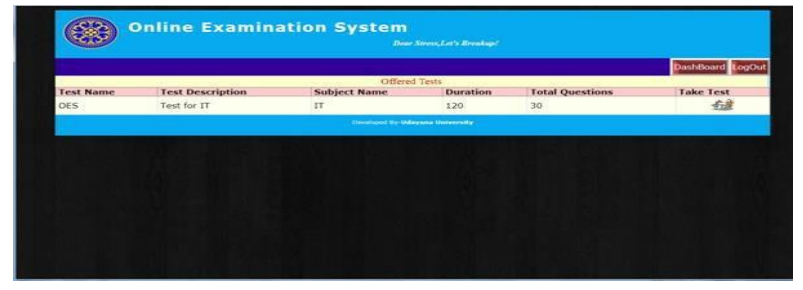

(a)

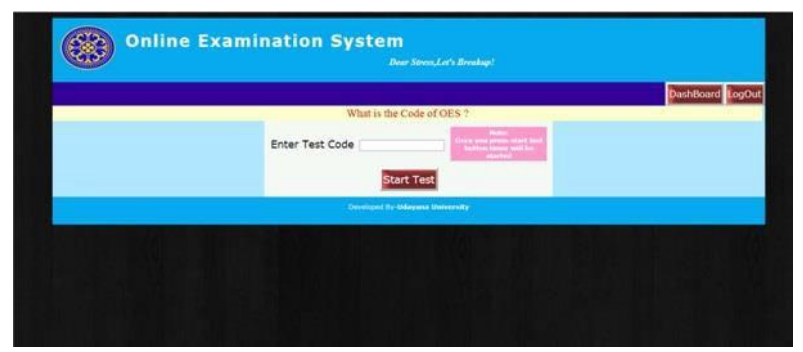

(b)

Fig. 5.Starting the examination Stage

If the test code is successfully entered, the next process is the examination stage. At this stage, the e-exam server starts sending questions to the e-exam terminals. Then, at the same time the e-exam terminal generates TAMEx data and starts sending it periodically to the e-exam server. Based on the eexam process carried out by the examinee, the e-exam mechanism is a standard process of the e-exam process generally, namely the delivery question mechanism by the eexam server and the sending of answers by e-exam terminal that continues from the first question to the last question or until the time the e-exam ends. However, in the e-exam system which is implementing TAMEx model, the e-exam terminal will send 2 types of data to the e-exam server, namely the answer data from the question of exam and TAMEx data. Based on this TAMEx data the system identifies the connection status of the e-exam server.

This research presents a system with two (2) scenarios, namely when there is no connection loss and if there is a connection loss during the examination process which is sequentially shown in Figure 6 and Figure 7.
Figure 6 shows a process that was in normal condition. Thera are no any connection disruptions occur. So, the examination process holds from the time the first question is received by the e-exam terminal until the e-exam notification information is complete. Each of which is shown in figures

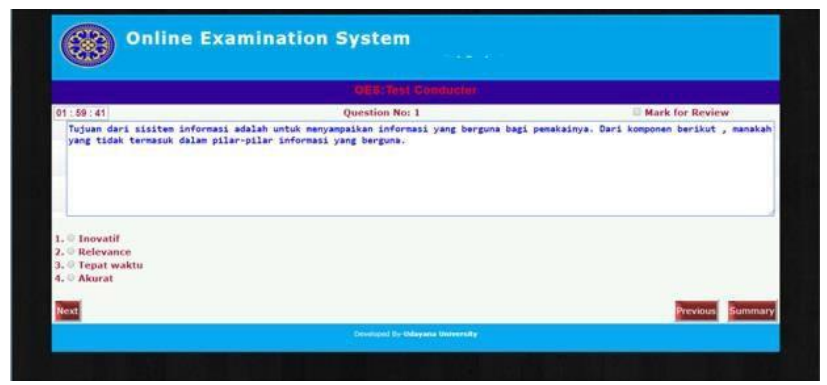

(a)

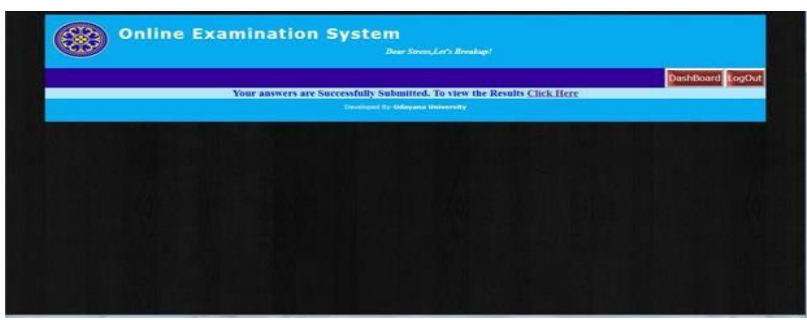

(b)

Fig; 6. Examination in no connection loss condition (a) The first question delivered; (b) Ending the examination stages

$6 a$ and $6 b$ respectively.

The system was tested for the occurrence of connection loss is shown in figure 7. This test is conducted to find out whether the system with this TAMEx model has succeeded in providing time compensation in the event of a connection loss experienced by the e-exam terminal automatically. The disconnection scenario was done by disconnecting the WiFi connection on the laptop that had been used as the e-exam terminal. The connection termination was done in a few minutes, then it had been reconnected.

Figure 7 shows the application display when the connection is disconnected (Figure 7a) and when the connection is re-connected (Figure 7b). From the Figure 7a, we can see the remaining test time is 01"42"41 (1 Hour 42 Minutes and 41 Seconds) while the laptop time is 2.22 PM. The test was done on September 11th, 2017. When the connection lost occur, the Next, Previous and Summary buttons did not work. The condition when the WiFi reconnected is shown in Figure $7 b$. From the picture can seen that the remaining time of the test 01 "42"24 (1 Hour 42 Minutes and 24 Seconds). While, the time on the laptop shows 2.23 PM. From the two pictures, there was about 17 seconds differences between the time when the connection was lost and when it was reconnected. Those condition occurs because when the laptop got an adequate WiFi signal strength it was about $20 \%$ of RSSI level, the system on the laptop directly sent the TAMEx data to the server. Then, the server reactivated the e-exam application of the e-exam 
terminal which had been locked. So, the time deviation was possibly caused by the computational and data transmission processes.

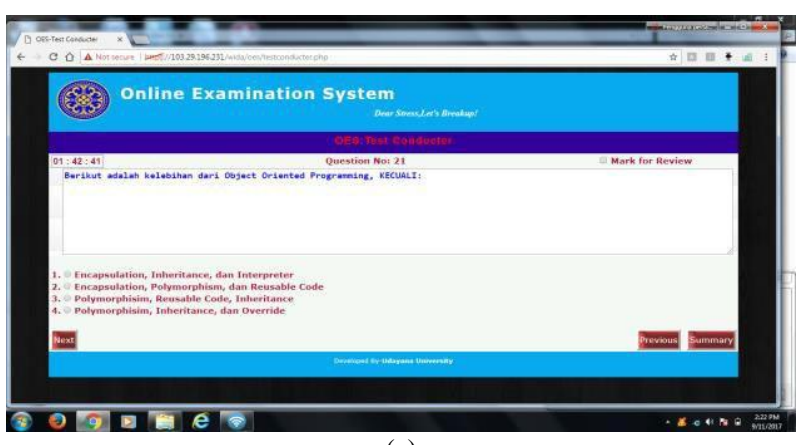

(a)

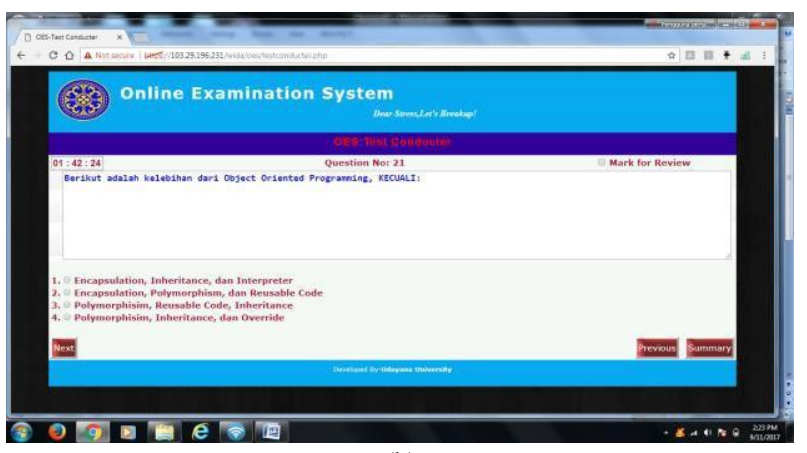

(b)

Fig. 7. The application when the connection loss occurred (a) Start the connection loss; (b) when reconnected

\section{B. Result}

As a result of this research, the following is discussed the recorded TAMEx data and the time compensation data given to the user or examinee. Table 1 is a snapshot of TAMEx data taken for testing by 5 users. As can be seen in table 1, there are some data fields recorded on the system that relate to the identity of user's, the the e-exam terminal device and the WLAN property received by the e-exam terminal.

Table 1 shows that each user accesses e-exam through different SSID. This means that the implementation of the TAMEx model which is using a laptop as an e-exam terminal strongly supports the mobility of e-exam participants. However, the use of the same SSID by several participants is very possible. Likewise, if the exam manager wants to restrict the access of participants from only certain SSIDs, it can be done by limiting authentication access only through a certain SSID.

Receive Signal Strength Indicator (RSSI) generated through the Netsh WLAN Interface command expressed in percent $(\%)$. In the TAMEx model, the e-exam terminal status is not connected when RSSI $<20 \%$. While, RSSI $\geq$ $20 \%$ the status is connected because at RSSI $=20 \%$ the service quality is in Roaming Thereshold condition [11]. MAC and IP address respectively provide information about the identity of the used e-exam terminal device and the location of the examinees accessing the e-exam. Whereas, Bitrate is the information about the available datarate on SSID.

Time is a field that informs the arrival time of TAMEx Data received on the server. Based on this arrival time, the server identifies connection disruptions experienced by the e-exam terminal. In this TAMEx Model application, the eexam terminal is set to generate and transmit TAMEx data every 5 seconds. If the time duration between data arrival and previous data is more than 5 seconds, the excess is indicated as loss time. For example, if the time duration between arrivals of the data is 10 seconds, the system will identify that there has been a loss connection for 5 seconds. Thus, the system needs to seek time compensation in accordance with the duration of the loss connection.

TABLE I

RECORDED TAMEX DATA

\begin{tabular}{|c|c|c|c|c|c|c|}
\hline user & SSID & $\begin{array}{c}\text { RSSI } \\
(\%)\end{array}$ & MAC & ipadd & $\begin{array}{l}\text { Bitrate } \\
\text { (Mbps) }\end{array}$ & time \\
\hline user1 & acara@unud & 99 & c8:3d:d4:9f:82:81 & 10.10 .18 .192 & 58 & 03:52:38 PM \\
\hline user1 & acara@unud & 99 & c8:3d:d4:9f:82:81 & 10.10 .18 .192 & 58 & 03:52:44 PM \\
\hline user1 & acara@unud & 87 & c8:3d:d4:9f:82:81 & 10.10 .18 .192 & 14.5 & 04:05:41 PM \\
\hline user1 & acara@unud & 99 & c8:3d:d4:9f:82:81 & 10.10 .18 .192 & 58 & 04:06:00 PM \\
\hline user1 & acara@unud & 99 & c8:3d:d4:9f:82:81 & 10.10 .18 .192 & 58 & 04:06:20 PM \\
\hline user2 & ElektroNET & 100 & $\mathrm{~d} 0: 53: 49: 15: 0 \mathrm{f}: 50$ & 192.168.5.109 & 54 & 04:04:50 PM \\
\hline user2 & ElektroNET & 100 & d0:53:49:15:0f:50 & 192.168.5.109 & 54 & 04:04:51 PM \\
\hline user2 & ElektroNET & 100 & d0:53:49:15:0f:50 & 192.168.5.109 & 54 & 04:05:30 PM \\
\hline user2 & ElektroNET & 100 & $\mathrm{~d} 0: 53: 49: 15: 0 \mathrm{f}: 50$ & 192.168.5.109 & 54 & 04:05:50 PM \\
\hline user2 & ElektroNET & 100 & d0:53:49:15:0f:50 & 192.168.5.109 & 54 & 04:05:52 PM \\
\hline user 3 & ElektroNET & 80 & $02: \mathrm{d} 0: \mathrm{f0}: 57: \mathrm{a} 6: 0 \mathrm{~b}$ & 192.168 .5 .110 & 54 & 04:57:21 PM \\
\hline user3 & ElektroNET & 80 & $02: \mathrm{d} 0: \mathrm{f0}: 57: \mathrm{a} 6: 0 \mathrm{~b}$ & 192.168 .5 .110 & 54 & 04:57:27 PM \\
\hline user3 & ElektroNET & 80 & $02: \mathrm{d} 0: \mathrm{f0}: 57: \mathrm{a} 6: 0 \mathrm{~b}$ & 192.168 .5 .110 & 54 & 04:57:31 PM \\
\hline user 3 & ElektroNET & 80 & $02: \mathrm{d} 0: \mathrm{f0}: 57: \mathrm{a} 6: 0 \mathrm{~b}$ & 192.168 .5 .110 & 54 & 04:57:37 PM \\
\hline user3 & ElektroNET & 80 & $02: \mathrm{d} 0: \mathrm{f0}: 57: \mathrm{a} 6: 0 \mathrm{~b}$ & 192.168 .5 .110 & 54 & 04:57:41 PM \\
\hline user 4 & @wifi.id & 90 & $54: 27: 1 \mathrm{e}: 0 \mathrm{e}: 56: 59$ & 192.168 .101 .1 & 72.2 & 04:02:00 PM \\
\hline user 4 & $@$ wifi.id & 90 & $54: 27: 1 \mathrm{e}: 0 \mathrm{e}: 56: 59$ & 192.168 .101 .1 & 72.2 & 04:02:05 PM \\
\hline user 4 & Q wifi.id & 90 & $54: 27: 1 \mathrm{e}: 0 \mathrm{e}: 56: 59$ & 192.168 .101 .1 & 72.2 & 04:02:10 PM \\
\hline user 4 & @wifi.id & 90 & $54: 27: 1 \mathrm{e}: 0 \mathrm{e}: 56: 59$ & 192.168.101.1 & 72.2 & 04:02:15 PM \\
\hline user 4 & @wifi.id & 88 & $54: 27: 1 \mathrm{e}: 0 \mathrm{e}: 56: 59$ & 127.0 .0 .1 & 72.2 & 04:04:49 PM \\
\hline user 5 & imissu@unggul & 90 & fo:03:8c:6d:4f: 15 & 192.168 .150 .1 & 72.2 & 04:14:33 PM \\
\hline user 5 & imissu@unggul & 90 & fo:03:8c:6d:4f: 15 & 192.168 .150 .1 & 72.2 & $04: 14: 44 \mathrm{PM}$ \\
\hline user5 & imissu@unggul & 90 & fo:03:8c:6d:4f: 15 & 192.168 .150 .1 & 72.2 & $04: 14: 45 \mathrm{PM}$ \\
\hline user5 & imissu@unggul & 90 & f0:03:8c:6d:4f:15 & 192.168 .150 .1 & 72.2 & 04:14:49 PM \\
\hline user 5 & imissu@unggul & 90 & f0:03:8c:6d:4f:15 & 192.168 .150 .1 & 72.2 & $04: 14: 54 \mathrm{PM}$ \\
\hline
\end{tabular}

TABLE II

RECORDED OF COMPENSATION TIME

\begin{tabular}{|c|c|c|c|}
\hline Student_name & Student_id & Test_id & $\begin{array}{c}\text { AddTime } \\
\text { Total } \\
\text { (seconds) }\end{array}$ \\
\hline User1 & 11 & 33 & 163 \\
\hline User2 & 6 & 33 & 0 \\
\hline User3 & 4 & 33 & 23 \\
\hline User4 & 7 & 33 & 102 \\
\hline User5 & 5 & 33 & 0 \\
\hline
\end{tabular}

Table 2 is a snapshot of the compensation time record table as the total time compensation given to each user. Can be seen in table 2, that User 2 and User 5 do not get time compensation, this means that both users did not experience connection problems during the inspection stage. In contrast to users 1, 3 and 4 users, each of them gets a compensation time of 163 seconds, 23 seconds and 102 seconds. The duration of this time compensation is in accordance with the 
total connection interruption experienced by each user. Thus, additional time is given to adapt to the signal quality conditions received by each e-exam terminal with a duration that corresponds to the connection loss experienced during theexamination stage.

\section{CONCLUSION AND FUTURE WORKS}

\section{A. Conclusion}

Development of Prototype e-exam applications using the TAMEx model can be done. The TAMEx Adapter is an application installed on a laptop carried by the examinee before being used as an e-exam terminal. This application serves to generate and send TAMEx data periodically to the e-exam server. TAMEx data containing components of user identity, e-exam terminal identity and WLAN property were successfully recorded in the database. The server then identifies the duration of the connection interruption based on the duration between the arrival time of the TAMEx data and the arrival time of the previous TAMEx data. Time compensation is given automatically at any time after the eexam terminal is reconnected due to a connection interruption. It is done by updating the information remaining time of the examination stage in the e-exam application. The total compensation time varies from one user to another because they experience a different duration of connection loss. This TAMEx model maintains the availability of e-exam by adapting the system with RSSI through time compensation only to users in accordance to the their experienched of connection loss.

\section{B. Future Works}

As a future work to improve this research there are several things that need to be done include:

1. Further research needs to be done to optimize the determination of the time period for generating and sending TAMEx data by e-exam terminal. This is needed to ensure the server's performance to record and its computing process to identify the duration of the connection loss that occurred.

2. Further research is also needed to determine with certainty the level of RSSI that is in application saturation conditions, so that the setting of the loss connection condition can be done well.

\section{ACKNOWLEDGMENT}

We would like to thank to our colleagues at Electrical Engginering Department, Faculty of Engineering, Udayana University which have provided greatly assisted the research. Many thank also to the Udayana University Research and Community Service Institute (LPPM) which has provided Research Grants on the skim of Unggulan Udayana.

\section{REFERENCES}

[1] G. Sukadarmika, R. S. Hartati, Linawati, and N. P. Sastra, "Introducing TAMEx model for availability of e-exam in wireless environment," 2018 Int. Conf. Inf. Commun. Technol. ICOIACT 2018, vol. 2018-Janua, pp. 163-167, 2018, doi: 10.1109/ICOIACT.2018.8350741.

[2] A. Sohail, Z. Ahmad, and I. Ali, "Analysis and measurement of Wi-Fi signals in indoor environment," Int. J. Adv. Eng. Technol., vol. 6, no. 2, pp. 678-687, 2013, doi: 10.1002/mrdd.

[3] Ç. Kurnaz, B. K. Engiz, and U. Köse, "Investigating the Effect of Number of Users on Signal Strength Level and Throughput for Wi-Fi System," no. December, 2017, doi: 10.1109/IDAP.2017.8090222.

[4] S. A. N and S. S. T. S. Vasavi, "Study on Techniques for Providing Enhanced Security During Online Exams," vol. 1, no. 1, pp. 32-37, 2012.

[5] M. Onyesolu, V. Ejiofor, M. Onyeizu, and D. Ugoh, "Enhancing Security in a Distributed Examination Using Biometrics and Distributed Firewall System," Int. J. Emerg. Technol. Adv. Eng., vol. 3, no. 9, p. 6, 2013.

[6] T. Ramu and T. Arivoli, "a Framework of Secure Biometric Based Online Exam Authentication: an Alternative To Traditional Exam," Int. J. Sci. Eng. Res., vol. 4, no. 11, pp. 52-60, 2013.

[7] Y. Sabbah, I. Saroit, and A. Kotb, "A Smart Approach for Bimodal Biometric Authentication in Home-Exams (SABBAH Model)," Biometrics Bioinforma., p. 13, 2012.

[8] S. J. Alotaibi, "Using biometrics authentication via fingerprint recognition in e-exams in e-learning environment," 4th Saudi Int. Conf., no. July, p. 10, 2010.

[9] M. Sarrayrih and M. Ilyas, "Challenges of Online Exam , Performances and problems for Online University Exam," Int. J. Comput. Sci., vol. 10, no. 1, pp. 439-443, 2013.

[10]E. C. Lo, "An Investigation of the Impact of Signal Strength on Wi-Fi Link Throughput through Propagation Measurement,” 2007.

[11]J. Bardwell, "Converting Signal Strength Percentage to dBm Values," WildPackets, Inc, no. November, pp. 1-12, 2002, doi: 20021217-MWP007. 